\title{
Advantages of targeting B cell receptor complex to treat B-cell derived autoimmune diseases and lymphomas
}

\section{Adem, Jemal}

2017-08

Adem , J , Eray , M , Eeva , J , Nuutinen , U \& Pelkonen , J 2017 , ' Advantages of targeting B cell receptor complex to treat B-cell derived autoimmune diseases and lymphomas ', Molecular Immunology , vol. 88 , pp. 135-137 . https://doi.org/10.1016/j.molimm.2017.05.023

http://hdl.handle.net/10138/297919

https://doi.org/10.1016/j.molimm.2017.05.023

publishedVersion

Downloaded from Helda, University of Helsinki institutional repository.

This is an electronic reprint of the original article.

This reprint may differ from the original in pagination and typographic detail.

Please cite the original version. 
Letter to the Editor

\section{A R T I C L E I N F O}

\section{Keywords:}

BCR

Rituximab

CD20

BAFF-R

Autoimmune diseases

Lymphoma

\begin{abstract}
A B S T R A C T
Antibodies produced by B-cells provide protection from infectious agents. However, impaired cell death signaling pathways in B-cells can lead to cancer, immunodeficiency or autoimmune diseases. B-cell signaling molecules such as CD20, CD19, Btk, and BAFF-R are targeted by therapeutic drugs and used to treat B-cell derived lymphomas or autoimmune diseases. Nevertheless, B-cells could develop resistance to these therapeutic drugs or the therapeutic drugs may have off-target effects. For instance, repeated rituximab (anti-CD20 antibody) treatment may lead to the loss of its target cell surface molecule, CD20. In addition, in B-cell malignancies, loss of CD19 expression has been observed. Another target molecule, Btk is expressed not only in B-cells but also in mast cells, macrophages, and dendritic cells. Thus, targeting Btk could negatively regulate the functions of innate immunity. The expression of BAFF-R is thought to be restricted to B-cells but it is also expressed on Tcells. Targeting BAFF-R, therefore, may lead to depletion of T-cells in addition to B-cells. B cell receptor (BCR) expression and signaling, however, are critically important for development, differentiation and survival of Bcells. Moreover, BCR is exclusively expressed on B-cells, which makes it an excellent target to avoid off-target effects.
\end{abstract}

B-lymphocytes (B-cells) are core components of adaptive immunity. The main functions of B-cells include antigen presentation and antibody production. However, defects in B-cell growth, development and selection can result in malignancy, immunodeficiency, and autoimmunity (Yanaba et al., 2008; Basso and Dalla-Favera, 2015; Ahn and Cunningham-Rundles, 2009). B cell receptor (BCR) is a transmembrane receptor protein located on the surface of B-cells. Signaling through BCR is required for normal progression of B-cell development. In humans, B-cells are produced in the bone marrow from hematopoietic precursor cells and they migrate to secondary lymphoid organs (periphery) where they encounter antigens. In the bone marrow, the VDJ recombination process produces BCR repertoires that are able to recognize a large array of antigens. In the periphery, B-cells can be activated by a variety of infectious agents through BCR. This phenomenon leads to the formation of germinal centers (GCs) (Pieper et al., 2013). In GCs, B-cells undergo somatic hypermutation (SHM), which increases BCR diversity and thereby increases chances of producing B-cells with high affinity to antigens. However, the SHM process can also lead to the production of autoreactive- B cells (Mackay and Rose, 2001).

The BCR complex consists of an antigen-binding subunit and a signaling subunit. The antigen binding subunit is a membrane-bound immunoglobulin (mIg) which lacks any signaling motif. The mIg part consists of two heavy and two light chains combined to each other by disulfide bonds. The signaling subunit consists of the accessory proteins CD79a (Ig $\alpha)$ and CD79b $(\operatorname{Ig} \beta)$ which transmit the activating signals to the celís interior. The recognition of specific antigens by the mIg, therefore, leads to the activation of a number of kinases such as Brutońs tyrosine kinase (Btk), phosphatidyl-inositol-3-kinase (PI3K) and extracellular signal-regulated kinases (ERKs) (Niiro and Clark, 2002; Treanor, 2012).

As we demonstrate in our previous work, the early activation of ERK1 and ERK2 following BCR stimulation results in short-term survival of GC Bcells. However, in the late phase, BCR stimulation leads to inhibition of ERK1 and ERK2, which correlates with cell death (Adem et al., 2015, Fig. 1). Interestingly, BCR-mediated apoptosis of GC B-cells is reversed by CD40 signaling (Adem et al., 2015, Fig. 4). These findings indicate that B-cells which overexpress ERK1 and ERK2 might bypass the selection process. Thus, B-cells which overexpress ERK1/2 and possess memory or plasma cell transcription signatures may transform into self-reactive memory B-cells or auto-antibody producing plasma cells. In addition, it is also possible that B cells which overexpress ERK1/2 but lack differentiation specific signatures may transform into lymphoma cells. Overexpression of Rasgrf-1 amplifies the Ras-ERK pathway in chronic lymphocytic leukemia cells, thereby enhancing B-cell survival. This indicates that malignant B-cells utilize ERKs for their survival (Liao et al., 2014). Moreover, changes in the activation of Ras-ERKs pathway may lead to autoimmune manifestations (Teodorovic et al., 2014). Thus, targeting ERKs may have therapeutic benefits against these B-cell derived diseases. However, because of the critical role of ERKs in various cellular functions, the use of ERK inhibitors can lead to severe cellular toxicity. It is, therefore, imperative to selectively target B-cells in order to avoid the side-effects which could result from off-target inhibition.

Rituximab is a chimeric monoclonal antibody which targets CD20, the cell surface differentiation antigen on B-cells. CD20 has a role in B-cell activation and differentiation. It is expressed in mature B-cells but not in hematological stem cells, pro-B cells, normal plasma cells or other tissues. This unique characteristic of CD20 expression makes it the suitable target to treat B-cell derived lymphomas or autoimmune diseases (Du et al., 2007). However, rituximab does not deplete autoreactive antibody-producing plasma cells, because these cells do not express CD20. Nevertheless, rituximab depletes B-cells, thereby decreasing the production of autoreactive antibody and malignant B-cells. The loss of CD20 expression, however, after repeated rituximab treatment may contribute to B-cells' resistance against rituximab (Haidar et al., 2003). A study on CD20 ${ }^{\prime-}$ (knockout) mouse has shown normal development and function in CD20 deficient B-cells (Uchida et al., 2004). This data further strengthen the fact that CD20 is not a critical cell surface molecule for the survival and development of B cells. CD19 is another pan B-cell marker used to target and deplete B-cell 
populations. However, different types of lymphomas lose CD19 expression (Masir et al., 2006).

Targeting signaling molecules or their receptors which have critical functions in B-cell development or survival helps to overcome drug resistance which results from the lack of CD20 or CD19 expression. As the majority of mature B-cell lymphoma express functional BCR, several small molecule inhibitors have been developed to inhibit a variety of kinases in the BCR pathway including Btk (Rickert, 2013). However, Btk is also expressed in several other hematopoietic lineages such as neutrophils, thrombocytes, mast cells, macrophages and dendritic cells (Brunner et al., 2005; Honda et al., 2012; Futatani et al., 2001). Inhibition of Btk, therefore, could compromise innate immunity. In this regard, the adverse events of treating chronic lymphocytic leukemia/lymphomas patients with Ibrutinib, a BTK inhibitor, include infections, neutropenia, and thrombocytopenia (Varma et al., 2016). Moreover, the lack of tissue specificity of Ibrutinib limits their utility. However, lymphoma patients respond to Ibrutinib.

BAFF-R is another surface molecule used to target B-cells and its expression is thought to be B-cell specific. Interestingly, BAFF-R is also expressed in T-cells subsets ( $\mathrm{Ng}$ et al., 2004). The elimination of B-cells through BAFF-R could also deplete T-cells populations, thereby negatively regulate Tcell-mediated immunity. Several studies have demonstrated the efficacy and safety of anti-BAFF agents such as tabalumab and atacicept. The data show considerable variability in response to BAFF-R/BAFF blockage. For instance, tabalumab did not provide the intended clinical efficacy in rheumatoid arthritis (Smolen et al., 2015) and multiple myeloma patients (Raje et al., 2017). Moreover, treatment with atacicept was well tolerated and no dose-limiting toxicity was observed in B-cell lymphoma patients, however, none of the subjects achieved complete or partial responses (Ansell et al., 2008). These poor clinical responses may be due to the substantial heterogeneity in the pathogenesis of autoimmune diseases/lymphomas.

Given the complexity of the pathogenesis of B-cell derived diseases, it is imperative to identify a potential target molecule that is B-cell specific and critical to cell survival. In this regard, BCR could be an excellent target to eliminate a malignant or autoreactive B-cells because BCR is required for the development and survival of B-cells. In $\lambda 5$ knockout mice, B-cell development was severely impaired (Miyazaki et al., 1999). In addition, conditional deletion of surface IgM in mature B-cell leads to rapid apoptosis, indicating that regardless of antigen encounter, signaling through BCR is necessary for the survival of B-cells (Lam et al., 1997). Therefore, it is less likely that BCR-dependent lymphomas/auto-reactive B-cells develop resistance by losing BCR expression because the loss of BCR compromises B-cell survival. As shown in mice study, inhibition of B cells through CD79b is much more effective than depletion of B-cells with the anti-CD20 antibody (Bruhl et al., 2015). In addition, anti-CD79b antibody induces B-cell anergy via downregulation of BCR components. This distinct feature of the anti-CD79b antibody shows that it also suppress the pathogenic immune responses in the absence of B cell depletion (Hardy et al., 2014). Furthermore, a clinical trial study shows that patients diagnosed with relapsed or refractory B-cell lymphoma responded to polatuzumab vedotin (an anti-CD79b drug conjugate) (Pfeifer et al., 2015), suggesting that BCR complex could be the suitable target to treat lymphomas.

Interestingly, the expression of CD79a and CD79b is restricted to B-cells and these co-receptors are not expressed in other healthy tissue and immune cells. Nonetheless, CD79a is found to be expressed in T-cell lymphoma (Chu and Arber, 2001; Hashimoto et al., 2002). Moreover, there is a case report indicating the co-existence of B-cell and T-cell lymphoma in some patients (Campidelli et al., 2007). Thus, the expression of CD79a in Tcell lymphoma in addition to B-cell lymphoma could be exploited as the therapeutic target to treat B-cell and T-cell lymphoma simultaneously, however, the dual presence of these diseases in a single patient is an exception.

Epstein-Barr Virus (EBV)-derived lymphoma cells may not retain dependency on BCR for their survival. Because EBV infection can rescue BCR ${ }^{-}$ (B-cell with no BCR expression) GC B cells from apoptosis (Mancao et al., 2005). Therefore, this finding indicates that targeting BCR complex could also have its limitation and should be considered according to the origin of the disease.

The mIg could also be used as a therapeutic target, for example, monoclonal antibody targeting epitope restricted to mIgM has anti-tumor effects (Welt et al., 2016). The anti-tumor effects of the anti-mIgM antibody, however, could not be extended to follicular lymphoma (FL), because FL originates from B-cells which have undergone class-switching to IgG (Aarts et al., 2002). Moreover, the anti-IgD antibody is efficient in depleting mature B cells (Nguyen et al., 2010). However, IgD might not be the best target to eliminate self-reactive memory B cells because these cells lack IgD expression. Idiotopes (Ids) are other mIg components that have been used as therapeutic targets against cancer (de Cerio et al., 2007). The limitations with this approach are the therapeutic antibodies needed to be individually customized and the hypervariable region associated Ids may be lost due to residual hypermutation activity in target B-cells.

Altogether, these data show that the BCR complex, either the mIg (constant domain) or CD79a/CD79b is the suitable therapeutic target for B-cell derived diseases. It might also be used as a target for patients who do not respond to B-cell specific immunotherapy such as anti-CD20 or anti-CD19 antibody. Thus, the anti-BCR antibody can be used as a cell-specific therapeutic agent against autoimmune diseases and lymphomas. Furthermore, we suggest that the anti-BCR antibody conjugated with a plant or bacterial-derived toxin could enhance therapeutic efficiency.

\section{Conflict of interest statement}

The authors declare no financial or commercial conflict of interest.

\section{Acknowledgments}

The research work was supported by grants from the research committee of the Kuopio University Hospital Catchment Area (State Research Funding), North-Savo cancer organization and Doctoral programme of Molecular Medicine of University of Eastern Finland.

\section{References}

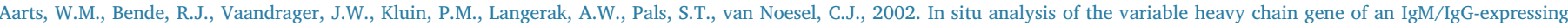
follicular lymphoma: evidence for interfollicular trafficking of tumor cells. Am. J. Pathol. 3, 883-891.

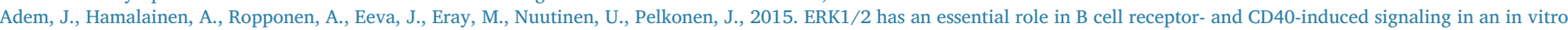
model of germinal center B cell selection. Mol. Immunol. 2 (Pt. B), 240-247.

Ahn, S., Cunningham-Rundles, C., 2009. Role of B cells in common variable immune deficiency. Expert Rev. Clin. Immunol. 5, 557-564.

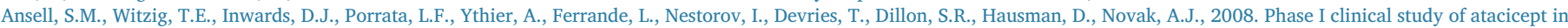
patients with relapsed and refractory B-cell non-Hodgkin's lymphoma. Clin. Cancer Res. 4, 1105-1110.

Basso, K., Dalla-Favera, R., 2015. Germinal centres and B cell lymphomagenesis. Nat. Rev. Immunol. 3, $172-184$.

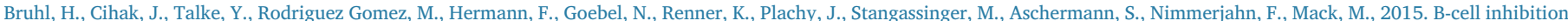
by cross-linking CD79b is superior to B-cell depletion with anti-CD20 antibodies in treating murine collagen-induced arthritis. Eur. J. Immunol. 3, 705-715.

Brunner, C., Muller, B., Wirth, T., 2005. Bruton's tyrosine kinase is involved in innate and adaptive immunity. Histol. Histopathol. 3, 945-955.
} 


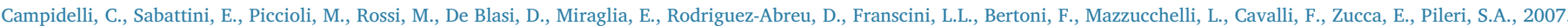
Simultaneous occurrence of peripheral T-cell lymphoma unspecified and B-cell small lymphocytic lymphoma. Report of 2 cases. Hum. Pathol. 5, 787-792.

Chu, P.G., Arber, D.A., 2001. CD79: a review. Appl. Immunohistochem. Mol. Morphol. 2, 97-106.

de Cerio, A.L., Zabalegui, N., Rodriguez-Calvillo, M., Inoges, S., Bendandi, M., 2007. Anti-idiotype antibodies in cancer treatment. Oncogene 25, 3594-3602.

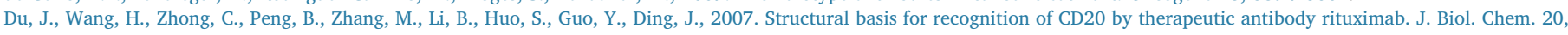
$15073-15080$.

Futatani, T., Watanabe, C., Baba, Y., Tsukada, S., Ochs, H.D., 2001. Bruton's tyrosine kinase is present in normal platelets and its absence identifies patients with X-linked agammaglobulinaemia and carrier females. Br. J. Haematol. 1, 141-149.

Haidar, J.H., Shamseddine, A., Salem, Z., Mrad, Y.A., Nasr, M.R., Zaatari, G., Bazarbachi, A., 2003. Loss of CD20 expression in relapsed lymphomas after rituximab therapy. Eur. J. Haematol. 5, 330-332.

Hardy, I.R., Anceriz, N., Rousseau, F., Seefeldt, M.B., Hatterer, E., Irla, M., Buatois, V., Chatel, L.E., Getahun, A., Fletcher, A., Cons, L., Pontini, G., Hertzberg, N.A., Magistrelli, G., Malinge, P., Smith, M.J., Reith, W., Kosco-Vilbois, M.H., Ferlin, W.G., Cambier, J.C., 2014. Anti-CD79 antibody induces B cell anergy that protects against autoimmunity. J. Immunol. 4, 1641-1650.

Hashimoto, M., Yamashita, Y., Mori, N., 2002. Immunohistochemical detection of CD79a expression in precursor T cell lymphoblastic lymphoma/leukaemias. J. Pathol. 3, 341-347.

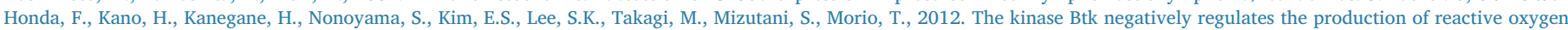
species and stimulation-induced apoptosis in human neutrophils. Nat. Immunol. 4, 369-378.

Lam, K.P., Kuhn, R., Rajewsky, K., 1997. In vivo ablation of surface immunoglobulin on mature B cells by inducible gene targeting results in rapid cell death. Cell 6, 1073-1083.

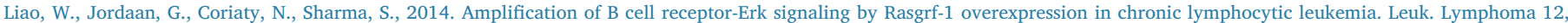
2907-2916.

Mackay, I.R., Rose, N.R., 2001. Autoimmunity and lymphoma: tribulations of B cells. Nat. Immunol. 9, 793-795.

Mancao, C., Altmann, M., Jungnickel, B., Hammerschmidt, W., 2005. Rescue of crippled germinal center B cells from apoptosis by Epstein-Barr virus. Blood 13, 4339-4344.

Masir, N., Marafioti, T., Jones, M., Natkunam, Y., Rudiger, T., Hansmann, M.L., Mason, D.Y., 2006. Loss of CD19 expression in B-cell neoplasms. Histopathology 3, $239-246$.

Miyazaki, T., Kato, I., Takeshita, S., Karasuyama, H., Kudo, A., 1999. Lambda5 is required for rearrangement of the Ig kappa light chain gene in pro-B cell lines. Int. Immunol. 8, 1195-1202.

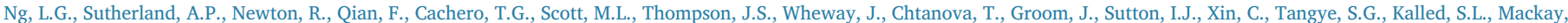
F., Mackay, C.R., 2004. B cell-activating factor belonging to the TNF family (BAFF)-R is the principal BAFF receptor facilitating BAFF costimulation of circulating T and B cells. J. Immunol. 2, 807-817.

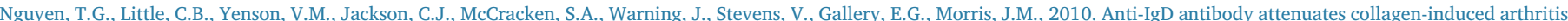
by selectively depleting mature B-cells and promoting immune tolerance. J. Autoimmun. 1, 86-97.

Niiro, H., Clark, E.A., 2002. Regulation of B-cell fate by antigen-receptor signals. Nat. Rev. Immunol. 12, 945-956.

Pfeifer, M., Zheng, B., Erdmann, T., Koeppen, H., McCord, R., Grau, M., Staiger, A., Chai, A., Sandmann, T., Madle, H., Dorken, B., Chu, Y.W., Chen, A.I., Lebovic, D., Salles, G.A., Czuczman, M.S., Palanca-Wessels, M.C., Press, O.W., Advani, R., Morschhauser, F., Cheson, B.D., Lenz, P., Ott, G., Polson, A.G., Mundt, K.E., Lenz, G., 2015. Anti-CD22 and antiCD79B antibody drug conjugates are active in different molecular diffuse large B-cell lymphoma subtypes. Leukemia 7, $1578-1586$.

Pieper, K., Grimbacher, B., Eibel, H., 2013. B-cell biology and development. J. Allergy Clin. Immunol. 4, 959-971.

Raje, N.S., Moreau, P., Terpos, E., Benboubker, L., Grzasko, N., Holstein, S.A., Oriol, A., Huang, S.Y., Beksac, M., Kuliczkowski, K., Tai, D.F., Wooldridge, J.E., Conti, I., Kaiser, C.J., Nguyen, T.S., Cronier, D.M., Palumbo, A., 2017. Phase 2 study of tabalumab, a human anti-B-cell activating factor antibody, with bortezomib and dexamethasone in patients with previously treated multiple myeloma. Br. J. Haematol. 5, 783-795.

Rickert, R.C., 2013. New insights into pre-BCR and BCR signalling with relevance to B cell malignancies. Nat. Rev. Immunol. 8, 578-591.

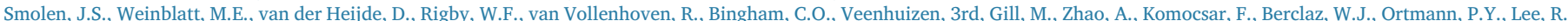
2015. Efficacy and safety of tabalumab, an anti-B-cell-activating factor monoclonal antibody, in patients with rheumatoid arthritis who had an inadequate response to methotrexate therapy: results from a phase III multicentre, randomised, double-blind study. Ann. Rheum. Dis. 8, 1567-1570.

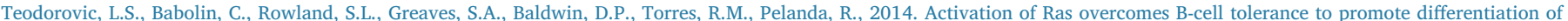
autoreactive B cells and production of autoantibodies. Proc. Natl. Acad. Sci. U. S. A. 27, E2797-806.

Treanor, B., 2012. B-cell receptor: from resting state to activate. Immunology 1, 21-27.

Uchida, J., Lee, Y., Hasegawa, M., Liang, Y., Bradney, A., Oliver, J.A., Bowen, K., Steeber, D.A., Haas, K.M., Poe, J.C., Tedder, T.F., 2004. Mouse CD20 expression and function. Int. Immunol. 1, 119-129.

Varma, G., Johnson, T.P., Advani, R.H., 2016. Bruton's tyrosine kinase inhibitors in chronic lymphocytic leukemia and lymphoma. Clin. Adv. Hematol. Oncol. 7, 543-554.

Welt, R.S., Welt, J.A., Kostyal, D., Gangadharan, Y.D., Raymond, V., Welt, S., 2016. Specificity and biologic activities of novel anti-membrane IgM antibodies. Oncotarget 46, $74701-74723$.

Yanaba, K., Bouaziz, J.D., Matsushita, T., Magro, C.M., St Clair, E.W., Tedder, T.F., 2008. B-lymphocyte contributions to human autoimmune disease. Immunol. Rev. 284-299.

Department of Clinical Microbiology, Institute of Clinical Medicine, University of Eastern Finland, Yliopistonranta 1C, 70210 Kuopio, Finland E-mail address: jemal.adem@uef.fi

Mine Eray

Department of Pathology (HUSLAB), Helsinki University Hospital, Helsinki, Finland

Jonna Eeva

Department of Hematology, Tampere University Hospital, Tampere, Finland

Ulla Nuutinen

Department of Clinical Microbiology, Institute of Clinical Medicine, University of Eastern Finland, Yliopistonranta 1C, 70210, Kuopio, Finland

Jukka Pelkonen"

Department of Clinical Microbiology, Institute of Clinical Medicine, University of Eastern Finland, Yliopistonranta 1C, 70210, Kuopio, Finland

Eastern Finland Laboratory Centre (IS LAB), Kuopio, Finland

Cancer Center of University of Eastern, Finland

* Corresoponding author. 